\title{
Weighted $L^{\infty}$-estimates for Bergman projections
}

\author{
by \\ José Bonet (Valencia), Miroslav Engliš (Praha) \\ and JARI TASKINEN (Helsinki)
}

\begin{abstract}
We consider Bergman projections and some new generalizations of them on weighted $L^{\infty}(\mathbb{D})$-spaces. A new reproducing formula is obtained. We show the boundedness of these projections for a large family of weights $v$ which tend to 0 at the boundary with a polynomial speed. These weights may even be nonradial. For logarithmically decreasing weights bounded projections do not exist. In this case we instead consider the projective description problem for holomorphic inductive limits.
\end{abstract}

1. Introduction. We consider the Bergman projections

$$
P_{\alpha} f(z):=C_{\alpha} \int_{\mathbb{D}} \frac{f(\zeta)\left(1-|\zeta|^{2}\right)^{\alpha}}{(1-z \bar{\zeta})^{2+\alpha}} d A(\zeta),
$$

where $C_{\alpha}:=\alpha+1, \alpha>-1, \mathbb{D} \subset \mathbb{C}$ is the open unit disc, and $d A$ is the normalized 2-dimensional Lebesgue measure on $\mathbb{D}$. Békollé characterized the weight functions $v: \mathbb{D} \rightarrow \mathbb{R}^{+}$for which $P_{\alpha}$ is bounded on the space $L_{v}^{p}(\mathbb{D})$, $1 \leq p<\infty$, in his work [2]: the weight $v$ has to satisfy a Muckenhoupt-type condition. Such a characterization in the case $p=\infty$ seems to be missing. The situation is more complicated, since for the constant weight no bounded projection exists. In this paper we prove quite general sufficient conditions for boundedness. These apply if $v$ behaves in a sense polynomially near the boundary of $\mathbb{D}$. For example, if the variation of $v$ is bounded on every hyperbolic ball with radius 1 , and if the radial behavior of $v$ is controlled, for some $b>0$, by

$$
\int_{0}^{1} \frac{K_{b}(r, s)}{v\left(s e^{i \theta}\right)} d s \leq \frac{C}{v(z)} \quad \text { for all } z=r e^{i \theta} \in \mathbb{D},
$$

2000 Mathematics Subject Classification: 47B38, 46A13.

Key words and phrases: Bergman projection, weighted estimate, projective description problem.

The research of Bonet was partially supported by MCYT and FEDER project BFM2001-2670 and AVCIT Grupo 03/050. The research of Taskinen was partially supported by the Academy of Finland project "Functional Analysis and Applications". 
where $K_{b}(r, s):=1 /(1-s)$ if $s \leq r$, and $K_{b}(r, s):=(1-s)^{b}(1-r)^{-b-1}$ if $s>r$, and the nonradial behaviour is controlled, for some $a>0$, by

$$
\frac{1}{v\left(e^{i \tau} z\right)} \leq C\left(\frac{|\tau|}{1-|z|}\right)^{a} \frac{1}{v(z)} \quad \text { for all } \tau \in[-\pi, \pi],|\tau| \geq(1-|z|) / 2,
$$

then $P_{\alpha}$ is bounded $L_{v}^{\infty} \rightarrow L_{v}^{\infty}$ for every $\alpha>a+b$. This is the result of Theorem 4 . Notice that for example $v(z):=(1-|z|)^{c}$ satisfies (2) for $0<c<b+1$.

In Section 2 we consider a large, new class of generalized Bergman projections $P_{F}$. A general reproducing formula is proven in Theorem 1. For suitably chosen examples we prove that $P_{F}$ is bounded on the spaces $L_{\beta}^{\infty}:=L_{v}^{\infty}$ with $v=(1-|z|)^{\beta}$, simultaneously for all $\beta$. Recall that none of the projections $P_{\alpha}$ has this property. (This result is also useful for applications to holomorphic inductive limits, which are defined in terms of some weight families.)

If the radial weight $v$ approaches 0 only at logarithmic speed, there does not exist any bounded projections from $L_{v}^{\infty}$ onto the corresponding holomorphic subspace $H_{v}^{\infty}$ (see for example [10]). However, it turns out that, even in the logarithmic case, a Bergman projection method combined with a cut-off argument is still useful enough to attack the so-called projective description problem for holomorphic inductive limits. This is explained in Section 4 .

Notations. We denote by $\mathbb{H}$ the shifted right half-plane $\{z \in \mathbb{C}: \operatorname{Re} z>$ $1 / 2\}$. By $C$ we denote a positive constant which may vary from place to place, but not in the same inequality. Similarly, $C_{\alpha}$ denotes a constant depending on $\alpha$, and so on. By $f(x) \sim g(x)$ we mean that there exists a positive constant $C$ independent of $x$ such that $C^{-1} f(x) \leq g(x) \leq C f(x)$ for all $x$.

For basics of the hyperbolic metric on $\mathbb{D}$ we refer to [11, Section 4].

By a weight we mean a strictly positive bounded continuous function $v: \mathbb{D} \rightarrow \mathbb{R}$. Concerning the behavior of weights, "polynomial" etc. weight refers to a polynomial of the function $1 /(1-|z|)$ rather than to a polynomial of $z$. Given a weight, we define $\|f\|_{v}:=\sup _{z \in \mathbb{D}}|f(z)| v(z)$ if $f$ is continuous, with "ess sup" replacing "sup" if $f$ is just measurable. We write $L_{v}^{\infty}:=$ $\left\{f: \mathbb{D} \rightarrow \mathbb{C}\right.$ measurable $\left.\mid\|f\|_{v}<\infty\right\}$, and denote by $C v$ (respectively, by $H_{v}^{\infty}$ or $H v$ ) the closed subspace consisting of continuous (resp. holomorphic) functions. The notation $L_{\beta}^{\infty}, \beta>-1$, stands for the space $L_{v}^{\infty}$ with $v(z):=$ $(1-|z|)^{\beta}$. By $C v_{0}$ and $H v_{0}$, respectively, we mean the closed subspaces of $C v$ and $H v$ consisting of functions such that $\lim _{|z| \rightarrow 1^{-}}|f(z)| v(z)=0$.

Finally, we recall basic facts on inductive limits of weighted spaces of continuous or holomorphic functions. If $\mathcal{V}=\left(v_{k}\right)_{k=1}^{\infty}$ is a decreasing sequence of weight functions defined on $\mathbb{D}$, the weighted inductive limits $\operatorname{ind}_{k} C v_{k}$ and $\operatorname{ind}_{k} H v_{k}$ are denoted by $\mathcal{V} C$ and $\mathcal{V} H$; they are the spaces $\bigcup_{k} C v_{k}$ and 
$\bigcup_{k} H v_{k}$ endowed with the finest locally convex topologies such that the canonical embeddings $C v_{k} \rightarrow \mathcal{V} C$ and $H v_{k} \rightarrow \mathcal{V} H$ are continuous.

The inductive limits $\mathcal{V}_{0} C$ and $\mathcal{V}_{0} H$ are defined in the same way using the subspaces $C\left(v_{k}\right)_{0}$ and $H\left(v_{k}\right)_{0}$ instead of $C v_{k}$ and $H v_{k}$.

In order to describe the topology of the weighted inductive limits, Bierstedt, Meise and Summers [5] associated with the sequence $\mathcal{V}$ the system $\bar{V}$ of all those weights $\bar{v}: \mathbb{D} \rightarrow] 0, \infty\left[\right.$ such that, for all $k$, the quotient $\bar{v} / v_{k}$ is bounded on $\mathbb{D}$. The corresponding projective hull $C \bar{V}$ (resp. $H \bar{V}$ ) is the locally convex space of all those continuous (resp. holomorphic) functions on $\mathbb{D}$ such that, for all $\bar{v} \in \bar{V}$,

$$
\|f\|_{\bar{v}}=\sup _{z \in \mathbb{D}} \bar{v}(z)|f(z)|<\infty,
$$

endowed with the canonical sup-seminorms.

The projective description problem asks if the topologies $H \bar{V}$ and $\mathcal{V} H$ coincide; the same question can be posed for the other pair of spaces as well. We refer to K. Bierstedt's survey [3] for further details and open problems about projective description. Our results in Section 4 complement the results for the polynomially decreasing weights in [4] and [9].

2. Exponential case. We introduce a large family of reproducing kernels and study the boundedness properties of the corresponding projection operators in this and the next section.

Recall that the mapping

$$
(z, \zeta) \mapsto \frac{1-z \bar{\zeta}}{1-|\zeta|^{2}}
$$

maps $\mathbb{D} \times \mathbb{D}$ onto $\mathbb{H}=\{z \in \mathbb{C}: \operatorname{Re} z>1 / 2\}$.

TheOREM 1. Let $F$ be a holomorphic function on $\mathbb{H}$ satisfying

$$
\begin{gathered}
\sup _{j=0,1,2, \ldots} \frac{1}{j !} \int_{1}^{\infty}\left|(x-1)^{j} F^{(j)}(x)\right| d x<\infty, \\
\gamma_{F}:=\int_{1}^{\infty} F(x) d x \neq 0,
\end{gathered}
$$

and

$$
\lim _{x \in \mathbb{R}, x \rightarrow \infty} x^{k+1} F^{(k)}(x)=0 \quad \forall k=0,1,2, \ldots
$$

Then for any $z \in \mathbb{D}$ and any holomorphic function $g$ on $\mathbb{D}$ for which the integral on the left hand side exists,

$$
\int_{\mathbb{D}} g(\zeta) F\left(\frac{1-z \bar{\zeta}}{1-|\zeta|^{2}}\right) \frac{d A(\zeta)}{\left(1-|\zeta|^{2}\right)^{2}}=\gamma_{F} g(z) .
$$


Proof. Passing to the polar coordinates $\zeta=r e^{i \theta}$, the hypothesis of the existence of the integral on the left hand side of (7) implies that this integral equals the repeated integral

$$
\int_{0}^{1} \int_{0}^{2 \pi} g\left(r e^{i \theta}\right) F\left(\frac{1-z r e^{-i \theta}}{1-r^{2}}\right) \frac{d \theta}{2 \pi} \frac{2 r d r}{\left(1-r^{2}\right)^{2}} .
$$

Observe that for any $0<r<1$ and any pair of functions $f(\zeta)=\sum_{j=0}^{\infty} f_{j} \zeta^{j}$ and $g(\zeta)=\sum_{j=0}^{\infty} g_{j} \zeta^{j}$ holomorphic in a neighbourhood of $r \overline{\mathbb{D}}$,

$$
\int_{0}^{2 \pi} g\left(r e^{i \theta}\right) f\left(r e^{-i \theta}\right) \frac{d \theta}{2 \pi}=\int_{0}^{2 \pi} \sum_{j, k=0}^{\infty} g_{j} f_{k} r^{j+k} e^{(j-k) i \theta} \frac{d \theta}{2 \pi}=\sum_{j=0}^{\infty} f_{j} g_{j} r^{2 j},
$$

the interchange of summation and integration being justified by the uniform convergence of the Taylor series on $r \overline{\mathbb{D}}$. Applying this, in particular, to $f(\zeta)=F\left(\frac{1-z \zeta}{1-r^{2}}\right)$, we see that the inner integral in (8) equals

$$
\left.\sum_{j=0}^{\infty} g_{j} r^{2 j} \frac{1}{j !} \frac{d^{j}}{d \zeta^{j}} F\left(\frac{1-z \zeta}{1-r^{2}}\right)\right|_{\zeta=0}=\sum_{j=0}^{\infty} g_{j} r^{2 j} \frac{1}{j !}\left(\frac{-z}{1-r^{2}}\right)^{j} F^{(j)}\left(\frac{1}{1-r^{2}}\right) .
$$

Performing the change of variable $\frac{1}{1-r^{2}}=x$, we thus obtain

$$
\text { (8) }=\int_{1}^{\infty} \sum_{j=0}^{\infty} g_{j} \frac{z^{j}}{j !}(1-x)^{j} F^{(j)}(x) d x .
$$

In view of the absolute convergence of the Taylor series $\sum_{j=0}^{\infty} g_{j} z^{j}$ and (4), the integration and summation can again be interchanged. We thus see that the left hand side of (7) equals

$$
\sum_{j=0}^{\infty} \gamma_{j} g_{j} z^{j}
$$

with

$$
\gamma_{k}=\frac{(-1)^{k}}{k !} \int_{1}^{\infty}(x-1)^{k} F^{(k)}(x) d x .
$$

However, integration by parts reveals that

$$
\begin{aligned}
\gamma_{k}-\gamma_{k-1} & =\left[\frac{(1-x)^{k}}{k !} F^{(k-1)}(x)\right]_{x=1}^{x=\infty} \\
& =\lim _{x \in \mathbb{R}, x \rightarrow \infty}(1-x)^{k} F^{(k-1)}(x) / k !=0
\end{aligned}
$$

in view of $(6)$. Thus

$$
\gamma_{k}=\gamma_{k-1}=\cdots=\gamma_{0}=\gamma_{F} \quad \text { for all } k,
$$

with $\gamma_{F}$ given by (5). This completes the proof. 
REMARK 1. In view of (9), condition (4) is fulfilled, in particular, whenever the derivative $F^{(j)}(x)$ does not change sign on the interval $1 \leq x<\infty$, for each $j$, and $\left|\gamma_{F}\right|<\infty$ (since one then has $(1 / j !) \int_{1}^{\infty}\left|(x-1)^{j} F^{(j)}(x)\right| d x=$ $\left|\gamma_{j}\right|=\left|\gamma_{F}\right|$ for all $j$ ). In particular, taking $F(z)=e^{-z}$ or $F(z)=z^{-2} e^{-z}$, we have the reproducing formulas

$$
f(z)=e \int_{\mathbb{D}} \frac{f(\zeta)}{\left(1-|\zeta|^{2}\right)^{2}} \exp \left(-\frac{1-z \bar{\zeta}}{1-|\zeta|^{2}}\right) d A(\zeta)
$$

and

$$
f(z)=C \int_{\mathbb{D}} \frac{f(\zeta)}{(1-z \bar{\zeta})^{2}} \exp \left(-\frac{1-z \bar{\zeta}}{1-|\zeta|^{2}}\right) d A(\zeta),
$$

for any $f$ holomorphic on $\mathbb{D}$ for which the integrals exist.

Given $F$ as in Theorem 1 we define the projection operator

$$
P_{F} f(z):=\frac{1}{\gamma_{F}} \int \frac{f(\zeta)}{\left(1-|\zeta|^{2}\right)^{2}} F\left(\frac{1-z \bar{\zeta}}{1-|\zeta|^{2}}\right) d A(\zeta) \quad \text { with } \gamma_{F}:=\int_{1}^{\infty} F(x) d x
$$

Of course, $P_{\alpha}=P_{F}$ with $F(z):=z^{-\alpha-2}$.

Proposition 1. Assume that $v(z):=w\left(|z|^{2}\right)$ is a radial weight such that the function $B(s)=-\log w\left(1-e^{-s}\right)$ satisfies

$$
B^{\prime},\left(B^{\prime \prime}\right)^{1 / 2}=O\left(e^{(1 / 4-\delta) s}\right) \quad \text { for some } \delta>0,
$$

and that

$$
\sum_{k=1}^{\infty} \frac{\varrho^{k}}{w(1-1 / k)} \leq C \frac{1}{w\left(\varrho^{2}\right)} \quad \forall \varrho \in(0,1)
$$

for some finite constant $C$. Then the projection $P_{F}, F(z)=e^{-z}$, is bounded on $L_{v}^{\infty}$.

Proof. If $\|f\|_{v} \leq 1$, then

$$
\left|P_{F} f(z)\right| \leq e \int_{\mathbb{D}} \frac{1}{w\left(|\zeta|^{2}\right)} \exp \left[-\operatorname{Re} \frac{1-z \bar{\zeta}}{1-|\zeta|^{2}}\right] \frac{d A(\zeta)}{\left(1-|\zeta|^{2}\right)^{2}}
$$

We have

$$
\int_{0}^{2 \pi} \exp \left[-\operatorname{Re} \frac{1-z r e^{i \theta}}{1-r^{2}}\right] \frac{d \theta}{2 \pi}=e^{-1 /\left(1-r^{2}\right)} I_{0}\left(\frac{|z| r}{1-r^{2}}\right),
$$

where $I_{0}$ is the modified Bessel function (see [1, Section 7.2.2]). Switching to polar coordinates and estimating the radial integral as in the proof of 
Theorem 1 , we obtain the bound $(\varrho:=|z|)$

$$
\begin{aligned}
\left|P_{F} f(z)\right| & \leq e \int_{0}^{1} \frac{1}{w(t)} I_{0}\left(\frac{\varrho \sqrt{t}}{1-t}\right) e^{-1 /(1-t)} \frac{d t}{(1-t)^{2}} \\
& =e \int_{1}^{\infty} \frac{1}{w(1-1 / x)} I_{0}(\varrho \sqrt{x(x-1)}) e^{-x} d x \\
& \leq e \int_{1}^{\infty} \frac{1}{w(1-1 / x)} I_{0}(\varrho x) e^{-x} d x .
\end{aligned}
$$

We need to show that this is $\leq C / w\left(\varrho^{2}\right)$.

By formula 7.13.1(5) in [1],

$$
I_{0}(x)=\frac{e^{x}}{\sqrt{x}}(1+O(1 / x)) \quad \text { as } x \rightarrow \infty .
$$

We now use Theorem 1 of Holland and Rochberg [7] as follows: we take $a(t):=e^{-t}, b(t):=1 / w(1-1 / t)$ there (the hypothesis (13) precisely ensures that this is possible), and we obtain

$$
\frac{1}{k !} \int_{1}^{\infty} \frac{1}{w(1-1 / x)} x^{k} e^{-x} d x \sim \frac{1}{w(1-1 / k)} .
$$

Consequently,

$$
\begin{aligned}
\int_{1}^{\infty} \frac{1}{w(1-1 / x)} I_{0}(\varrho x) e^{-x} d x & \leq C \int_{1}^{\infty} \frac{1}{w\left(1-\frac{1}{x}\right)} \frac{e^{\varrho x}}{\sqrt{x}} e^{-x} d x \\
& =C \sum_{k=0}^{\infty} \frac{\varrho^{k}}{k !} \int_{1}^{\infty} \frac{1}{w(1-1 / x)} x^{k-1 / 2} e^{-x} d x \\
& \leq C^{\prime} \sum_{k=0}^{\infty} \frac{\varrho^{k}}{w(1-1 / k)} \\
& \leq \frac{C^{\prime \prime}}{w\left(\varrho^{2}\right)} \text { by }(14) . \text { - }
\end{aligned}
$$

In the next result we show that the projections $P_{F}$ with $F(z)=z^{-\alpha} e^{-z}$, $\alpha>1$, are bounded simultaneously for every polynomial weight $v(z)=$ $(1-|z|)^{\beta}, \beta>0$. (These weights do not satisfy the hypothesis of the preceding theorem.)

Some more projections $P_{F}$ with this property will be exhibited in the next section. However, the method of proof is completely different there.

Theorem 2. Let $\beta>0, \alpha>1$. Then the projection $P_{F}$ corresponding to $F(z)=z^{-\alpha} e^{-z}$ is bounded on $L_{\beta}^{\infty}:=L_{\left(1-|z|^{2}\right)^{\beta}}^{\infty}$. 
Proof. Let $\|f\|_{\left(1-|z|^{2}\right)^{\beta}} \leq 1$. Then (with $C_{F}:=\left|\gamma_{F}\right|^{-1}$ )

$$
\begin{aligned}
\left|P_{F} f(z)\right| & \leq C_{F} \int_{\mathbb{D}}\left|f(\zeta) F\left(\frac{1-z \bar{\zeta}}{1-|\zeta|^{2}}\right)\right| \frac{d A(\zeta)}{\left(1-|\zeta|^{2}\right)^{2}} \\
& \leq C_{F} \int_{\mathbb{D}}\left(\frac{1}{1-|\zeta|^{2}}\right)^{\beta}\left|\frac{1-\bar{\zeta} z}{1-|\zeta|^{2}}\right|^{-\alpha} e^{-\operatorname{Re} \frac{1-z \bar{\zeta}}{1-|\zeta|^{2}}} \frac{d A(\zeta)}{\left(1-|\zeta|^{2}\right)^{2}} \\
& =C_{F} \int_{0}^{1} \int_{0}^{2 \pi}\left(1-r^{2}\right)^{-\beta}\left|\frac{1}{1-r^{2}}-\frac{r|z| e^{i \theta}}{1-r^{2}}\right|^{-\alpha} e^{-\frac{1}{1-r^{2}}+\frac{\operatorname{Re} r|z| e^{i \theta}}{1-r^{2}}} \frac{d \theta}{2 \pi} \frac{2 r d r}{\left(1-r^{2}\right)^{2}} \\
& =C_{F} \int_{1}^{\infty} \int_{0}^{2 \pi} x^{\beta}|x-| z\left|\sqrt{x(x-1)} e^{i \theta}\right|^{-\alpha} e^{-x} e^{|z| \sqrt{x(x-1)} \cos \theta} \frac{d \theta}{2 \pi} d x \\
& =C_{F} \int_{1}^{\infty} \int_{0}^{\pi} x^{\beta-\alpha}\left|1-R e^{i \theta}\right|^{-\alpha} e^{-x} e^{R x \cos \theta} \frac{d \theta}{\pi} d x,
\end{aligned}
$$

where we have made the changes of variable $\zeta=r e^{-i \theta}|z| / \bar{z}$ and $x=$ $1 /\left(1-r^{2}\right)$, and $R:=|z| \sqrt{1-1 / x}$; note that $0 \leq R<|z|<1$. We need to show that this double integral is $\leq C\left(1-|z|^{2}\right)^{-\beta}$, for some $C$ independent of $z$. The integral is clearly uniformly bounded when $z$ ranges in a compact subset of $\mathbb{D}$; we can thus assume that $|z|>1 / 2$. Easy estimates also show that the integral over $1 \leq x \leq 2$ stays bounded as $|z| \nearrow 1$; thus it is enough to deal only with the integral over $2<x<\infty$. However, for $x$ in this range and $|z|>1 / 2$ we have $R>1 / 2 \sqrt{2}$; also,

$$
1-\cos \theta=2 \sin ^{2} \frac{\theta}{2} \geq \frac{2}{\pi^{2}} \theta^{2}
$$

since $(\sin \xi) / \xi \geq\left(\sin \frac{\pi}{2}\right) / \frac{\pi}{2}=2 / \pi$ for $\xi \in(0, \pi / 2)$ by convexity. Thus

$$
\begin{aligned}
\left|1-R e^{i \theta}\right|^{2} & =1-2 R \cos \theta+R^{2}=(1-R)^{2}+2 R(1-\cos \theta) \\
& \geq(1-R)^{2}+\frac{1}{\sqrt{2}} \cdot \frac{2}{\pi^{2}} \theta^{2} \geq \frac{\sqrt{2}}{\pi^{2}}\left[(1-R)^{2}+\theta^{2}\right] \\
& \geq \frac{1}{\sqrt{2} \pi^{2}}[(1-R)+\theta]^{2}
\end{aligned}
$$

Since, further,

$$
\begin{aligned}
1-R & =(1-|z|)+|z|\left[1-\sqrt{1-\frac{1}{x}}\right] \geq(1-|z|)+|z|\left[1-\sqrt{1-\frac{1}{x}+\frac{1}{4 x^{2}}}\right] \\
& =(1-|z|)+\frac{|z|}{2 x} \geq(1-|z|)+\frac{1}{4 x},
\end{aligned}
$$

we see that 


$$
\left|1-R e^{i \theta}\right| \geq \frac{1}{q}\left[(1-|z|)+\frac{1}{x}+\theta\right]
$$

where $q=4 \sqrt[4]{2} \pi$. Since, as noted above, $R<|z|$, we therefore obtain

$$
\begin{aligned}
\int_{2}^{\infty} \int_{0}^{\pi} x^{\beta-\alpha}\left|1-R e^{i \theta}\right|^{-\alpha} & e^{-x} e^{R x \cos \theta} \frac{d \theta}{\pi} d x \\
& \leq q^{\alpha} \int_{2}^{\infty} \int_{0}^{\pi} x^{\beta-\alpha} e^{-x}[(1-|z|)+1 / x+\theta]^{-\alpha} e^{|z| x} \frac{d \theta}{\pi} d x \\
& =q^{\alpha} \int_{2}^{\infty} x^{\beta-\alpha} e^{-(1-|z|) x} \int_{0}^{\pi}[1-|z|+1 / x+\theta]^{-\alpha} \frac{d \theta}{\pi} d x .
\end{aligned}
$$

Making the substitution $\eta:=\theta / B$ shows that, for any $B>0$,

$$
\int_{0}^{\pi}(B+\theta)^{-\alpha} d \theta=B^{1-\alpha} \int_{0}^{\pi / B}(1+\eta)^{-\alpha} d \eta \leq B^{1-\alpha} \int_{0}^{\infty}(1+\eta)^{-\alpha} d \eta=\frac{B^{1-\alpha}}{\alpha-1}
$$

since $\alpha>1$. Thus we can continue our estimate with

$$
\begin{aligned}
& \leq \frac{q^{\alpha}}{(\alpha-1) \pi} \int_{2}^{\infty} x^{\beta-\alpha} e^{-(1-|z|) x}[(1-|z|)+1 / x]^{1-\alpha} d x \\
& =\frac{q^{\alpha}}{(\alpha-1) \pi} \int_{2}^{\infty} x^{\beta-1} e^{-(1-|z|) x}[1+(1-|z|) x]^{1-\alpha} d x \\
& =\frac{q^{\alpha}}{(\alpha-1) \pi}\left(\frac{1}{1-|z|}\right)^{\beta} \int_{2(1-|z|)}^{\infty} y^{\beta-1} e^{-y}(1+y)^{1-\alpha} d y \\
& \leq \frac{q^{\alpha}}{(\alpha-1) \pi} c_{\alpha, \beta}\left(\frac{1}{1-|z|}\right)^{\beta},
\end{aligned}
$$

where

$$
c_{\alpha, \beta}:=\int_{0}^{\infty} \frac{y^{\beta-1}}{(1+y)^{\alpha-1}} e^{-y} d y<\infty
$$

and we have made the change of variable $y:=(1-|z|) x$. This completes the proof.

REMARK 2. The following can be shown by similar arguments: for $\beta>0$, $P_{F}$ maps $L_{\left(1-|z|^{2}\right)^{\beta}}^{\infty}$ into $L_{\left(1-|z|^{2}\right)^{\beta-\alpha+1}}^{\infty}$ if $\alpha<1$, and into $L_{\left(1-|z|^{2}\right)^{\beta} /\left|\log \left(1-|z|^{2}\right)\right|}^{\infty}$ if $\alpha=1$. For $\beta=0, P_{F}$ maps the ordinary $L^{\infty}$ into $L_{1 /|\log (1-|z|)|}^{\infty}, L_{1 /|\log (1-|z|)|^{2}}^{\infty}$ and $L_{\left(1-|z|^{2}\right)^{1-\alpha}}^{\infty}$ if $\alpha>1, \alpha=1$ and $\alpha<1$, respectively; and for $\beta<0$, $P_{F}$ maps $L_{\left(1-|z|^{2}\right)^{\beta}}^{\infty}$ into the ordinary $L^{\infty}$ if $\alpha>\beta+1$, into $L_{1 /\left|\log \left(1-|z|^{2}\right)\right|}^{\infty}$ if $\alpha=\beta+1$, and into $L_{\left(1-|z|^{2}\right)^{\beta-\alpha+1}}^{\infty}$ if $\alpha<\beta+1$. 
Unfortunately it seems that the use of the projections $P_{F}$ is more restricted than perhaps expected. We have for example the following negative result.

THEOREM 3. Let $F$ be any function as in Theorem $1, v$ a radial weight on $\mathbb{D}$ vanishing at the boundary, and assume that $P_{F}$ is bounded on $L_{v}^{\infty}$. Then

$$
\liminf _{t / 1} \frac{\log v(t)}{\log (1-t)}<\infty
$$

Hence, if $v$ is also decreasing, then it decays at most polynomially as $|z| \nearrow 1$ :

$$
\exists A \exists t_{0} \forall t>t_{0}: \quad v(t) \geq(1-t)^{A} .
$$

In particular, $P_{F}$ is not bounded on $L_{v}^{\infty}$ for $v(z)=e^{-\left(1-|z|^{2}\right)^{-\beta}}, \beta>0$, for any $F$.

Proof. For $1 / 2<r<1$, define

$$
f_{r}(z):=e^{-i \theta(z, r)} / v(z) \in L_{v}^{\infty},
$$

where $\theta(\zeta, r) \in[0,2 \pi]$ is the argument of the complex number

$$
F\left(\frac{1-r \bar{\zeta}}{1-|\zeta|^{2}}\right)
$$

Clearly, $f_{r}$ has the property $\left\|f_{r}\right\|_{v}=1$. Thus

$$
\left\|P_{F}\right\|_{v \rightarrow v} \geq\left\|P_{F} f_{r}\right\|_{v} \geq\left|P_{F} f_{r} v(r)\right| .
$$

Define further $(1-r \bar{\zeta}) /\left(1-|\zeta|^{2}\right)=: \Phi_{r}(\zeta)$ and

$$
D_{r}:=\left\{\zeta \in \mathbb{D}\left|1-\frac{1-r}{2} \leq\right| \zeta\left|\leq 1-\frac{1-r}{4},\right| \arg (\zeta) \mid \leq \frac{1-r}{4}\right\} .
$$

Then (with $C_{F}:=\left|\gamma_{F}\right|^{-1}$ )

$$
\begin{aligned}
\left|P_{F} f_{r}(r)\right| & =C_{F} \int_{\mathbb{D}} \frac{1}{v(\zeta)}\left|F\left(\Phi_{r}(\zeta)\right)\right| \frac{d A(\zeta)}{\left(1-|\zeta|^{2}\right)^{2}} \\
& \geq \frac{C_{F}}{\sup _{D_{r}} v} \int_{D_{r}}\left|F\left(\Phi_{r}(\zeta)\right)\right| \frac{d A(\zeta)}{\left(1-|\zeta|^{2}\right)^{2}}
\end{aligned}
$$

Write for a moment $\zeta=x+y i$; a routine calculation of the Jacobian shows that

$$
\frac{d A\left(\Phi_{r}(\zeta)\right)}{d A(\zeta)}=\frac{r^{2}}{\left(1-|\zeta|^{2}\right)^{2}}+\frac{2 r(x-r)}{\left(1-|\zeta|^{2}\right)^{3}} .
$$

Since $x-r \sim 1-r$ and $1-|\zeta|^{2} \sim 1-r$ for $\zeta \in D_{r}$, it follows that

$$
d A\left(\Phi_{r}(\zeta)\right) \sim \frac{d A(\zeta)}{\left(1-|\zeta|^{2}\right)^{2}} \quad \text { on } D_{r} .
$$


Therefore for some constant $C^{\prime}>0$ (independent of $F$ and $r$ ),

$$
\left|P_{F} f_{r}(r)\right| \geq \frac{C_{F}}{\sup _{D_{r}} v} C^{\prime} \int_{D_{r}}\left|F\left(\Phi_{r}(\zeta)\right)\right| d A\left(\Phi_{r}(\zeta)\right)=\frac{C^{\prime} C_{F}}{\sup _{D_{r}} v} \int_{\Phi_{r}\left(D_{r}\right)}|F| d A .
$$

Thus from (15) we get

$$
\left\|P_{F}\right\| \geq C^{\prime} C_{F} \frac{v(r)}{\sup _{D_{r}} v} \int_{\Phi_{r}\left(D_{r}\right)}|F| d A .
$$

We now claim that the intersection $Q:=\bigcap_{1 / 2<r<1} \Phi_{r}\left(D_{r}\right)$ has positive measure. Indeed, we have

$$
\Phi_{r}\left(\frac{1+r}{2}\right)=\frac{4+2 r}{3+r}<\frac{3}{2} \text { and } \Phi_{r}\left(\frac{3+r}{4}\right)=\frac{4(4+r)}{7+r}>\frac{16}{7},
$$

so, by continuity, $\Phi_{r}\left(D_{r} \cap \mathbb{R}\right)$ always contains the interval $[3 / 2,16 / 7]$. On the other hand, consider the mapping

$$
d_{r}(x, y):=\Phi_{r}\left(\left(\frac{1+r}{2}+x \frac{1-r}{4}\right) e^{i y(1-r) / 4}\right)
$$

which maps the rectangle $0 \leq x \leq 1,-1 \leq y \leq 1$ onto $\Phi_{r}\left(D_{r}\right)$. One more tedious but routine calculation reveals that

$$
\nabla \Phi_{r}(x, 0)=\left[\begin{array}{cc}
\frac{(2-x)^{2} r^{2}+\left(12-x^{2}-4 x\right) r+8(x+2)}{(2-x)^{2}(6+2 r+(1-r) x)^{2}} & 0 \\
0 & \frac{r(2+2 r+(1-r) x)}{(2-x)(6+2 r+(1-r) x)}
\end{array}\right] .
$$

Straightforward estimates show that both diagonal elements are positive, bounded and bounded away from zero uniformly as $x \in[0,1]$ and $r \in$ $(1 / 2,1)$. It therefore follows that $\Phi_{r}\left(D_{r}\right)$ always contains also some small neighbourhood (independent of $r$ ) in $\mathbb{C}$ of, say, the subinterval $[7 / 4,2]$ of $[3 / 2,16 / 7]$. This establishes the claim.

Consequently, setting $C_{F}^{\prime \prime}:=\int_{Q}|F| d A>0$, it transpires from (16) that

$$
\left\|P_{F}\right\| \geq C_{F}^{\prime \prime} C^{\prime} C_{F} \frac{v(r)}{\sup _{D_{r}} v},
$$

so that

$$
\frac{v(r)}{\sup _{D_{r}} v} \leq \frac{\left\|P_{F}\right\|}{C_{F}^{\prime \prime} C^{\prime} C_{F}}=: c_{F}<\infty, \quad \text { by hypothesis. }
$$

Hence, for each $r \in(1 / 2,1)$, there exists $\varrho(r) \in[(1+r) / 2,(3+r) / 4]$ such that

$$
\frac{v(r)}{v(\varrho(r))} \leq c_{F} ;
$$

that is,

$$
\log \frac{1}{v(\varrho(r))} \leq \log \frac{1}{v(r)}+\log c_{F} .
$$


Iteration gives

$$
\log \frac{1}{v\left(\varrho^{k}(r)\right)} \leq \log \frac{1}{v(r)}+k \log c_{F}, \quad k=1,2,3, \ldots
$$

On the other hand, $(1-\varrho(r)) /(1-r) \in[1 / 4,1 / 2]$ for any $r$, so

$$
\log \frac{1}{1-\varrho^{k(r)}} \geq \log \frac{1}{1-r}+k \log 2 .
$$

Since the left hand side of (17) is positive for $k$ large enough, we can divide to get

$$
\frac{-\log v\left(\varrho^{k}(r)\right)}{-\log \left(1-\varrho^{k}(r)\right)} \leq \frac{-\log v(r)+k \log c_{F}}{-\log (1-r)+k \log 2} \rightarrow \frac{\log c_{F}}{\log 2}<\infty \quad \text { as } k \rightarrow \infty .
$$

Thus

$$
\liminf _{t / 1} \frac{-\log v(t)}{-\log (1-t)}<\infty
$$

which proves the first part of the theorem.

If $v$ is in addition decreasing, then for any $t \in\left[\varrho^{k}(r), \varrho^{k+1}(r)\right]$ we have

$$
v(t) \geq v\left(\varrho^{k+1}(r)\right) \text { and } 1-t \leq 1-\varrho^{k}(r) .
$$

Hence, for these $t$,

$$
\frac{-\log v(t)}{-\log (1-t)} \leq \frac{-\log v\left(\varrho^{k+1}(r)\right)}{-\log \left(1-\varrho^{k}(r)\right)} \leq \frac{-\log v(r)+(k+1) \log c_{F}}{-\log (1-r)+k \log 2},
$$

and it follows that even

$$
\limsup _{t / 1} \frac{-\log v(t)}{-\log (1-t)} \leq \limsup _{k \rightarrow \infty} \frac{-\log v(r)+(k+1) \log c_{F}}{-\log (1-r)+k \log 2}=\frac{\log c_{F}}{\log 2} .
$$

Defining $A:=\frac{\log c_{F}}{\log 2}+1$, it therefore transpires that there exists $t_{0} \in(0,1)$ such that for all $t>t_{0}$,

$$
\frac{-\log v(t)}{-\log (1-t)} \leq A
$$

or $v(t) \geq(1-t)^{A}$. This completes the proof.

3. Polynomial case. In this section we consider weights which tend to 0 polynomially at the boundary. Békollé characterized the boundedness of $P_{\alpha}$ for such weights on $L_{v}^{p}$ for $1 \leq p<\infty$. The characterization involved some Muckenhoupt-type conditions for the weights. On the other hand, in the radial case it is known that $P_{\alpha}$ is bounded on $L_{\beta}^{\infty}$ if $\alpha+1>\beta>0$. So we consider the case of the spaces $L_{v}^{\infty}$ with nonradial weights here.

It may be difficult to give a complete characterization for the boundedness of $P_{\alpha}$, since, for the constant weight, no bounded projection exists. We give instead some sufficient conditions. In general there will be different 
conditions for the radial and nonradial directions. The former will be rather accurate whereas the latter are quite loose.

Given $b>0$ define, for all $0<s, r<1, K_{b}(r, s):=1 /(1-s)$ if $s \leq r$, and

$$
K_{b}(r, s):=\frac{(1-s)^{b}}{(1-r)^{b+1}} \quad \text { if } s>r .
$$

TheOrem 4. Assume that there exist constants $a, b, C>0$ such that the weight $v$ satisfies

(i) the radial condition

$$
\int_{0}^{1} \frac{K_{b}(r, s)}{v\left(s e^{i \theta}\right)} d s \leq \frac{C}{v(z)} \quad \text { for all } z=r e^{i \theta} \in \mathbb{D},
$$

(ii) the angular condition

$$
\frac{1}{v\left(e^{i \tau} z\right)} \leq C\left(\frac{|\tau|}{1-|z|}\right)^{a} \frac{1}{v(z)}
$$

for all $\tau \in[-\pi, \pi],|\tau| \geq(1-|z|) / 2$, and

(iii) the local condition: there exists a $C>0$ such that for every hyperbolic ball $D \subset \mathbb{D}$ with radius at most 1 ,

$$
\inf _{z \in D} v(z) \geq C \sup _{z \in D} v(z) .
$$

The projection $P_{\alpha}$ is then bounded $L_{v}^{\infty} \rightarrow L_{v}^{\infty}$ for every $\alpha>a+b$. Also $P_{F}: L_{v}^{\infty} \rightarrow L_{v}^{\infty}$ is bounded if $F$ satisfies, for an $\alpha>a+b$,

$$
|F(z)| \leq C|z|^{-2-\alpha}
$$

for all $z \in \mathbb{H}$.

Clearly, $v(z):=\left(1-|z|^{2}\right)^{\beta}, \beta>0$, satisfies the assumptions of Theorem 4 with any $a>0, b>\beta-1$. On the other hand, $F(z)=e^{-z^{\gamma}}$ or $F(z)=$ $z^{-2} e^{-z^{\gamma}}, 0<\gamma<1$, satisfy (22) for all $\alpha$, hence, $P_{F}: L_{\beta}^{\infty} \rightarrow L_{\beta}^{\infty}$ is bounded for every $\beta$. More examples are presented at the end of this section.

The proof is based on a standard division of $\mathbb{D}$ into sets which essentially are hyperbolic balls with constant radius. So, for every $n \in \mathbb{N}$ and $m \in$ $M_{n}:=\left\{1,2,3, \ldots, 2^{n}\right\}$ define

$$
\begin{aligned}
& D_{n, m}:=\left\{z=r e^{i \theta} \in \mathbb{D} \mid 1-2^{-n} \leq r \leq 1-2^{-n-1}\right. \\
&\text { and } \left.(m-1) 2^{-n} \leq \theta / 2 \pi \leq m 2^{-n}\right\} .
\end{aligned}
$$

For each $n$ and $m$ fix the point $\lambda_{n, m}:=\left(1-2^{-n}\right) e^{2 \pi i m 2^{-n}} \in D_{n, m}$. We will show that under the assumptions of our theorem, the assumptions of the following lemma are satisfied, and hence Theorem 4 follows. Moreover, the lemma provides our most general sufficient condition. 
For every $N \in \mathbb{N}, M \in M_{N}$ and $n \in \mathbb{N}$, we denote by $\nu:=\nu(N, M, n)$ the positive integer closest to $M 2^{-N} 2^{n}$. (The purpose of this definition is that, given $n \neq N$, the number $e^{2 \pi i \nu 2^{-n}}$ is as close as possible to $e^{2 \pi i M 2^{-N}}$, i.e. $\lambda_{N, M}$ and $\lambda_{n, \nu}$ have nearly the same arguments.) Moreover, given $N \in \mathbb{N}$, $M \in M_{N}, n \in \mathbb{N}$ and $m \in M_{n}$, we define

$$
\mu:=\mu(N, M, n, m):=\min \left(|m-\nu|,\left|m-\nu-2^{n}\right|\right) .
$$

Lemma 1. Assume that there exists a strictly positive constant $C$ such that $v$ satisfies the following two conditions:

$$
C \inf _{\zeta \in D_{n, m}} v(\zeta) \geq \sup _{\zeta \in D_{n, m}} v(\zeta) \quad \text { for all } n, m,
$$

and, for all $N$ and $M \in M_{N}$,

$$
\begin{aligned}
\sum_{n<N} \sum_{m \in M_{n}} \frac{1}{\mu^{2+\alpha}} & \frac{1}{v\left(\lambda_{n, m}\right)}+\sum_{n \geq N} \sum_{m: \mu \leq 2^{n-N}} \frac{1}{v\left(\lambda_{n, m}\right)} 2^{(-n+N)(2+\alpha)} \\
& +\sum_{n \geq N} \sum_{m: 2^{n-N} \leq \mu \leq 2^{n}} \frac{1}{\mu^{2+\alpha}} \frac{1}{v\left(\lambda_{n, m}\right)} \leq \frac{C}{v\left(\lambda_{N, M}\right)} .
\end{aligned}
$$

Then $P_{\alpha}$ is bounded $L_{v}^{\infty} \rightarrow L_{v}^{\infty}$. Also $P_{F}: L_{v}^{\infty} \rightarrow L_{v}^{\infty}$ is bounded if $F$ satisfies (22).

Proof. Assume that $f \in L_{v}^{\infty}$ with $\|f\|_{v} \leq 1$. Let $z \in \mathbb{D}$ and let $N$ and $M$ be such that $z \in D_{N, M}$. We may assume $|z| \geq 3 / 4$. We estimate

$$
\begin{aligned}
\left|P_{\alpha} f(z)\right| & \leq C \int_{\mathbb{D}} \frac{|f(\zeta)|\left(1-|\zeta|^{2}\right)^{\alpha}}{|1-z \bar{\zeta}|^{2+\alpha}} d A(\zeta) \\
& \leq C \sum_{n, m} \int_{D_{n, m}} \frac{1}{v(\zeta)} \frac{\left(1-|\zeta|^{2}\right)^{\alpha}}{|1-z \bar{\zeta}|^{2+\alpha}} d A(\zeta) .
\end{aligned}
$$

By (25), we can here bound $1 / v(\zeta)$ by $C / v\left(\lambda_{n, m}\right)$ for $\zeta \in D_{n, m}$, for all $n$ and $m$. Moreover, $\left(1-|\zeta|^{2}\right)^{\alpha} \leq C\left(1-\left|\lambda_{n, m}\right|^{2}\right)^{\alpha} \leq C^{\prime} 2^{-n \alpha}$ for such $\zeta$. We consider three cases.

1. Let $n<N$. We always have

$$
\left|\frac{1-z \bar{\zeta}}{z /|z|-\zeta /|\zeta|}\right| \geq \frac{1}{2}
$$

since $|z| \geq 3 / 4$. Moreover, since $\zeta \in D_{n, m}$ and $2^{-n}(\nu-1) \leq \theta /(2 \pi) \leq$ $2^{-n}(\nu+1)$, we have

$$
\left|\frac{z}{|z|}-\frac{\zeta}{|\zeta|}\right| \geq C 2^{-n} \min \left(|m-\nu|,\left|m-\nu-2^{n}\right|\right)=C 2^{-n} \mu .
$$


This and (28) imply

$$
\frac{1}{|1-z \bar{\zeta}|^{2+\alpha}} \leq C \mu^{-2-\alpha} 2^{n(2+\alpha)} .
$$

The contribution of these terms in (27) is thus bounded by

$$
\sum_{n<N, m \in M_{n}} \frac{m\left(D_{n, m}\right)}{v\left(\lambda_{n, m}\right)} \frac{2^{-n \alpha} 2^{n(2+\alpha)}}{\mu^{2+\alpha}} \leq \sum_{n<N, m \in M_{n}} \frac{1}{v\left(\lambda_{n, m}\right)} \frac{1}{\mu^{2+\alpha}},
$$

and by (26), this is bounded by $C / v(z)$.

2. Assume $n \geq N$ and $\mu \leq 2^{n-N}$. Then $1-|z| \leq|1-z \bar{\zeta}|$ and hence

$$
\frac{1}{|1-z \bar{\zeta}|^{2+\alpha}} \leq C \frac{1}{(1-|z|)^{2+\alpha}} \leq C^{\prime} 2^{N(2+\alpha)} .
$$

Hence, these terms in (27) are bounded by

$$
\begin{aligned}
\sum_{n \geq N} \sum_{m: \mu \leq 2^{n-N}} \frac{1}{v\left(\lambda_{n, m}\right)} m & \left(D_{n, m}\right) 2^{N(2+\alpha)} 2^{-n \alpha} \\
& \leq \sum_{n \geq N} \sum_{m: \mu \leq 2^{n-N}} \frac{1}{v\left(\lambda_{n, m}\right)} 2^{(-n+N)(2+\alpha)},
\end{aligned}
$$

and we conclude as in case 1 .

3. In the case $n \geq N$ and $2^{n-N} \leq \mu \leq 2^{n}$ we again have (29), and we proceed as in case 1 .

Proof of Theorem 4. We show that the assumptions of Theorem 4 imply the assumptions of Lemma 1. Clearly (25) follows from (21).

As for (26), let $N$ and $M$ be arbitrary, and $z:=\lambda_{N, M}=\left(1-2^{-N}\right) e^{2 \pi i M 2^{-N}}$. First, given $n$, we have $\left|\lambda_{n, m}-\lambda_{n, \nu}\right| \leq C \mu 2^{-n}$ for all $m \in M_{n}$. Hence, (20) implies for all $m \in M_{n}$,

$$
\frac{1}{v\left(\lambda_{n, m}\right)} \leq C\left(\frac{\left|\lambda_{n, m}-\lambda_{n, \nu}\right|}{1-\left|\lambda_{n, \nu}\right|}\right)^{a} \frac{1}{v\left(\lambda_{n, \nu}\right)} \leq C^{\prime} \mu^{a} \frac{1}{v\left(\lambda_{n, \nu}\right)}
$$

The terms with $n<N$ in (26) are thus estimated by (since $a-2-\alpha<-1$ by assumption)

$$
\begin{aligned}
\sum_{n<N, m \in M_{n}} \frac{1}{\mu^{2+\alpha}} & \frac{1}{v\left(\lambda_{n, m}\right)} \leq C \sum_{n<N, m \in M_{n}} \mu^{a-2-\alpha} \frac{1}{v\left(\lambda_{n, \nu}\right)} \\
& \leq C^{\prime} \sum_{n<N} \frac{1}{v\left(\lambda_{n, \nu}\right)} \leq C^{\prime \prime} \sum_{n<N} \frac{m\left(I_{n}\right)}{\left(1-\left|\lambda_{n, \nu}\right|\right) v\left(\lambda_{n, \nu}\right)},
\end{aligned}
$$

where $I_{n}:=\left[1-2^{-n}, 1-2^{-n-1}\right]$. 
For $n \geq N, \mu \leq 2^{n-N}$ we again use (33):

$$
\begin{aligned}
\sum_{n \geq N} \sum_{m: \mu \leq 2^{n-N}} \frac{1}{v\left(\lambda_{n, m}\right)} 2^{(-n+N)(2+\alpha)} & \leq \sum_{n \geq N} \sum_{m: \mu \leq 2^{n-N}} \frac{1}{v\left(\lambda_{n, \nu}\right)} \mu^{a} 2^{(-n+N)(2+\alpha)} \\
& \leq C \sum_{n \geq N} \frac{1}{v\left(\lambda_{n, \nu}\right)} 2^{(-n+N)(1+\alpha-a)} .
\end{aligned}
$$

Since $n \geq N$ and $\alpha-a>b$, this is bounded by a constant times

$$
\sum_{n \geq N} \frac{1}{v\left(\lambda_{n, \nu}\right)} 2^{(-n+N)(b+1)} \leq C \sum_{n \geq N} m\left(I_{n}\right) \frac{\left(1-\left|\lambda_{n, \nu}\right|\right)^{b}}{\left(1-\left|\lambda_{N, \nu}\right|\right)^{b+1}} \frac{1}{v\left(\lambda_{n, \nu}\right)} .
$$

Finally, for $n \geq N, \mu \leq 2^{n-N}$ we similarly obtain

$$
\begin{aligned}
\sum_{n \geq N} \sum_{2^{n-N} \leq \mu \leq 2^{n}} \frac{1}{\mu^{2+\alpha}} \frac{1}{v\left(\lambda_{n, m}\right)} & \leq C \sum_{n \geq N} \sum_{2^{n-N} \leq \mu \leq 2^{n}} \frac{1}{v\left(\lambda_{n, \nu}\right)} \mu^{a-\alpha-2} \\
& \leq C^{\prime} \sum_{n \geq N} \frac{1}{v\left(\lambda_{n, \nu}\right)} 2^{(-n+N)(1+\alpha-a)}
\end{aligned}
$$

and we end up with the same bound as in (35).

Now the right hand side of (34) is a discretized version of the integral $\int_{0}^{r} K(r, s) / v\left(s e^{i \theta}\right) d s$ with $\theta:=2 \pi i M 2^{-N}$; the same is true for (35) and $\int_{r}^{1} K(r, s) / v\left(s e^{i \theta}\right) d s$, respectively. (Approximate $1 / v\left(s e^{i \theta}\right)$ by $1 / v\left(\lambda_{n, \nu}\right)$ if $s \in I_{n}$, and so on, and replace the integral by a sum; notice that $e^{2 \pi i \nu 2^{-n}}$ is very close to $e^{2 \pi i M 2^{-N}}$ because of the definition of $\nu$.) Hence, the sum of (34) and (35) can be bounded by a constant times $\int_{0}^{1} K(r, s) / v(s) d s$. The theorem thus follows from (19).

We give one more variant of Theorem 4 .

Proposition 2. If $v$ is a continuously differentiable weight satisfying (20) and (21) and moreover, for all $0<r<1$ and all $0 \leq \theta \leq 2 \pi$,

$$
\begin{aligned}
& \frac{1}{v\left(s e^{i \theta}\right)(1-s)} \leq C \frac{\partial}{\partial s} \frac{1}{v\left(s e^{i \theta}\right)} \quad \text { for all } s \leq r, \text { and } \\
& \frac{(1-s)^{b}}{v\left(s e^{i \theta}\right)} \leq-C \frac{\partial}{\partial s}\left(\frac{(1-s)^{b+1}}{v\left(s e^{i \theta}\right)}\right) \quad \text { for all } s \geq r,
\end{aligned}
$$

then the assumptions of Theorem 4 are satisfied, and $P_{\alpha}$ is thus bounded on $L_{v}^{\infty}$ for $\alpha>a+b$.

Notice that since $C>0$, the weights satisfying (36) must thus be decreasing on every line segment from 0 to $e^{i \theta}$. We remark that condition (37) implies

$$
\frac{1}{v\left(s e^{i \theta}\right)(1-s)} \geq C^{\prime} \frac{\partial}{\partial s} \frac{1}{v\left(s e^{i \theta}\right)} \quad \text { for all } s \geq r
$$


where $C^{\prime}:=b+1-C^{-1} \geq 0$. Conversely, if (38) holds, then also (37) is true for every $b>C^{\prime}-1$ (with $C:=\left(b+1-C^{\prime}\right)^{-1}$ ). These claims can be verified by performing the differentiation on the right hand side of (37).

Proof of Proposition 2. Integrate (36) and (37) to obtain (19).

This form of the sufficient conditions is interesting, since it is stable under products of weights:

Proposition 3. If $v$ and $w$ are weights satisfying the assumptions of Proposition 2, then vw satisfies them with $2 a$ and $2 b+1$ instead of $a$ and $b$, respectively.

Proof. Conditions (20) and (21) are clear, with $2 a$ replacing $a$ in (20). Concerning the other conditions, assume that $v$ and $w$ satisfy (36) and (37). Then

$$
\begin{aligned}
\frac{1}{v\left(s e^{i \theta}\right) w\left(s e^{i \theta}\right)}(1-s) \\
\quad=\frac{1}{2} \frac{1}{v\left(s e^{i \theta}\right)\left(w\left(s e^{i \theta}\right)(1-s)\right)}+\frac{1}{2} \frac{1}{w\left(s e^{i \theta}\right)\left(v\left(s e^{i \theta}\right)(1-s)\right)} \\
\leq C \frac{1}{v\left(s e^{i \theta}\right)} \frac{\partial}{\partial s} \frac{1}{w\left(s e^{i \theta}\right)}+C \frac{1}{w\left(s e^{i \theta}\right)} \frac{\partial}{\partial s} \frac{1}{v\left(s e^{i \theta}\right)} \\
\leq C \frac{\partial}{\partial s} \frac{1}{v\left(s e^{i \theta}\right) w\left(s e^{i \theta}\right)} \quad \text { for all } s \leq r .
\end{aligned}
$$

Moreover, for $s \geq r$ we get

$$
\begin{aligned}
\frac{(1-s)^{2 b+1}}{v\left(s e^{i \theta}\right) w\left(s e^{i \theta}\right)} \leq & -C \frac{(1-s)^{b+1}}{v\left(s e^{i \theta}\right)} \frac{\partial}{\partial s}\left(\frac{(1-s)^{b+1}}{w\left(s e^{i \theta}\right)}\right) \\
& -C \frac{(1-s)^{b+1}}{w\left(s e^{i \theta}\right)} \frac{\partial}{\partial s}\left(\frac{(1-s)^{b+1}}{v\left(s e^{i \theta}\right)}\right) \\
= & -C \frac{\partial}{\partial s}\left(\frac{(1-s)^{2 b+2}}{v\left(s e^{i \theta}\right) w\left(s e^{i \theta}\right)}\right) .
\end{aligned}
$$

EXAmple 1. Assume that $h:[0,2 \pi] \rightarrow \mathbb{R}$ is Lipschitz-continuous, $|h(\theta)-h(\tau)| \leq C|\theta-\tau|$ for all $\theta$ and $\tau$, and satisfies $h(0)=h(2 \pi)$ and $h(\theta)>0$ for all $\theta$. We claim that the weight

$$
v(z):=\left(1-|z|^{2}\right)^{h(\theta)}, \quad \text { where } z:=r e^{i \theta},
$$

satisfies the conditions of Theorem 4 for any $a>\max _{\theta} h(\theta)$ and $b=$ $\max _{\theta} h(\theta)-1$. Moreover, if $h$ is continuously differentiable, we claim that $v$ even satisfies the conditions of Proposition 2. If $h$ is not assumed continuous, the consequences may be quite dramatic, as shown in Example 2 below. 
Checking that $v$ satisfies (19) as claimed is trivial, since for each fixed angle, $v$ is equivalent to $(1-|z|)^{c}$ for some $0<c \leq \max _{\theta} h(\theta)$. The same is true for (36) and (37) in the differentiable case. Concerning (20), the Lipschitz-continuity of $h$ implies

$$
\frac{v(z)}{v\left(e^{i \tau} z\right)}=\frac{(1-|z|)^{h(\theta)}}{(1-|z|)^{h(\theta+\tau)}} \leq \frac{(1-|z|)^{h(\theta)}}{(1-|z|)^{h(\theta)+C|\tau|}}=e^{-C|\tau| \log (1-|z|)} .
$$

If

$$
\frac{1}{2}(1-|z|) \leq|\tau| \leq \frac{1}{|\log (1-|z|)|},
$$

then the right hand side of (40) is clearly bounded. On the other hand, $|\tau| /(1-|z|)$ in (20) is bounded from below, hence (20) follows for these $\tau$. If

$$
|\tau| \geq \frac{1}{|\log (1-|z|)|}
$$

then we set $\varepsilon:=a-\max _{\theta} h(\theta)>0$, and (41) implies

$$
|\tau|^{a}>C_{\varepsilon}(1-|z|)^{\varepsilon} .
$$

On the other hand, trivially

$$
\frac{1}{v\left(e^{i \tau} z\right)} \leq \frac{C}{(1-|z|)^{\max (\theta)}} \leq \frac{C^{\prime}}{(1-|z|)^{\max h(\theta)}} \frac{1}{v(z)} .
$$

Combining (42) and (43) gives

$$
\frac{1}{v\left(e^{i \tau} z\right)} \leq C \frac{|\tau|^{a}}{(1-|z|)^{\max h(\theta)+\varepsilon}} \frac{1}{v(z)}=C \frac{|\tau|^{a}}{(1-|z|)^{a}} \frac{1}{v(z)} .
$$

Finally, (21) is true, since for every $D$ as in (21) we have

$$
\inf _{z \in D}(1-|z|) \geq C \sup _{z \in D}(1-|z|) .
$$

EXAMPLE 2. We construct an example of a weight which fails even the weakest of the above conditions, and moreover, for which none of the Bergman projections $P_{\alpha}$ is bounded. To this end we define $\left.\left.h:\right] 0,2 \pi\right] \rightarrow \mathbb{R}^{+}$ by setting $h(\theta):=1$ if $\theta \in[\pi, 2 \pi], h\left(2 \pi 2^{-n}\right):=2$ if $n \in \mathbb{N}$ is even, and $h\left(2 \pi 2^{-n}\right):=1$ if $n$ is odd. We extend $h$ piecewise linearly for other values of $\theta \in] 0, \pi\left[\right.$. We then define $\widetilde{v}$ as in $(39), \widetilde{v}(z):=(1-|z|)^{h(\theta)}$ for $z=r e^{i \theta}$. But since this weight is not continuous on $[0,1[\subset \mathbb{D}$, we make it continuous as follows. Define the subset $\Lambda \subset \mathbb{D}$ (which is otherwise unimportant for the forthcoming calculations) by

$$
\Lambda:=\left\{z=x+i y \mid 0<x<1,0<y<e^{-1 /(1-x)}\right\} .
$$

Define $v(z):=\widetilde{v}(z)$ if $z \notin \Lambda$, and extend $v$ in an arbitrary way (for example using Tietze's theorem) to $\Lambda$ in such a way that it becomes continuous. 
Given $\alpha>-1$ we show that $P_{\alpha}$ is not bounded on $L_{v}^{\infty}$. So let an arbitrary odd $n \in \mathbb{N}$ be given, and consider $z=\left(1-2^{-n}\right) e^{-i 2 \pi 2^{-n}}$. Define the function

$$
f_{z}(\zeta):=\frac{e^{-i \theta(z, \zeta)}}{v(\zeta)} \quad \text { where } \quad \theta(z, \zeta):=\arg \left((1-z \bar{\zeta})^{2+\alpha}\right)
$$

which belongs to $L_{v}^{\infty}$ with $\|f\|_{v}=1$. Moreover, define the set

$$
\begin{aligned}
& E_{n, 2}:=\left\{\zeta=\varrho e^{i \tau} \in \mathbb{D} \mid 1-2^{-n} \leq \varrho \leq 1-2^{-n-1}\right. \\
& \text { and } \left.\frac{3}{2} \cdot 2^{-n} \leq \frac{\tau}{2 \pi} \leq 2 \cdot 2^{-n}\right\} \subset D_{n, 2} \text {. }
\end{aligned}
$$

The area of $E_{n, 2}$ is clearly at least $C 2^{-2 n}$. For $\zeta \in E_{n, 2}$ we have $h(\tau) \geq 3 / 2$ (recall $n$ is odd), hence, $v(\zeta) \leq 2^{-3 n / 2}$. So we can estimate

$$
\begin{aligned}
\left|P_{\alpha} f_{z}(z)\right| & =C\left|\int_{D_{n, m}} \frac{f_{z}(\zeta)\left(1-|\zeta|^{2}\right)^{\alpha}}{(1-z \bar{\zeta})^{2+\alpha}} d A(\zeta)\right| \\
& \geq C^{\prime} \int_{E_{n, m}} \frac{(1-|\zeta|)^{\alpha}}{(1-|z|)^{2+\alpha}} \frac{1}{v(\zeta)} d A(\zeta) \\
& \geq C^{\prime \prime} \int_{E_{n, m}} \frac{2^{-n \alpha}}{2^{-n(2+\alpha)}} 2^{3 n / 2} d A(\zeta) \geq C^{\prime \prime} 2^{3 n / 2} .
\end{aligned}
$$

On the other hand, $h(\arg z)=1$ by definitions, so, $v(z)=2^{-n}$. Hence, $\left\|P_{\alpha} f\right\|_{v} \geq\left|P_{\alpha} f(z)\right| v(z) \geq C 2^{n / 2}$ and $P_{\alpha}$ cannot be bounded.

We remark that weights constructed using discontinuous boundary values play a central role in the space of the paper [6]. That example is completely based on the nonexistence of (any) continuous projections from an $L^{\infty}$-type space onto its subspace of analytic functions.

Example 3. Consider weights of the form

$$
v(z):=\left(1-|z|^{2}\right)^{\alpha}\left(1-x^{2}\right)^{\beta}
$$

where $\alpha$ and $\beta$ are constants, $\alpha>0, \beta \geq 0$ and $z:=x+i y \in \mathbb{D}$. To check that they satisfy the conditions (36) and (37) of Proposition 2 we may assume $s \in[1 / 2,1[$. After elementary calculations we find that

$$
\begin{aligned}
\frac{\partial}{\partial s} \frac{1}{v\left(s e^{i \theta}\right)}= & \frac{1}{v\left(s e^{i \theta}\right)} \frac{1}{1-s^{2}} \frac{1}{1-(s \cos \theta)^{2}} \\
& \cdot(2 s)\left(\beta\left(1-s^{2}\right)(\cos \theta)^{2}+\alpha\left(1-(s \cos \theta)^{2}\right)\right)
\end{aligned}
$$




$$
\begin{aligned}
& \geq \frac{1}{v\left(s e^{i \theta}\right)} \frac{1}{1-s^{2}} \frac{1}{1-(s \cos \theta)^{2}} \cdot(2 s)\left(\alpha\left(1-(s \cos \theta)^{2}\right)\right) \\
& \geq C \frac{1}{v\left(s e^{i \theta}\right)} \frac{1}{1-s} .
\end{aligned}
$$

One can prove (37) in the same way using (38). Checking (21) is left to the reader. As for (20), write $z=r e^{i \theta}$ with $\theta \in[-\pi, \pi]$. Then (20) is equivalent to

$$
1-r \cos (\tau+\theta) \geq \frac{1}{C^{1 / \beta}}\left(\frac{1-r}{|\tau|}\right)^{a / \beta}(1-r \cos \theta) .
$$

Notice that the factor

$$
\frac{1}{C^{1 / \beta}}\left(\frac{1-r}{|\tau|}\right)^{a / \beta}
$$

can always be chosen smaller than any fixed positive constant. Moreover, we use the existence of a constant $c>1$ such that

$$
1-c s^{2} \leq \cos s \leq 1-s^{2} / c
$$

for every $s \in[-3 \pi / 2,3 \pi / 2]$.

(i) Assume that $|\tau| \leq|\theta| / 2$. Then $|\theta| / 2 \leq|\tau+\theta| \leq 3|\theta| / 2$, so by (51), the left hand side of (49) is at least

$$
\begin{aligned}
\min _{|\theta| / 2 \leq t \leq 3|\theta| / 2}(1-r \cos t) & \geq \min _{|\theta| / 2 \leq t \leq 3|\theta| / 2}\left(1-r\left(1-\frac{t^{2}}{c}\right)\right) \\
& =1-r+\frac{r \theta^{2}}{4 c} .
\end{aligned}
$$

On the right hand side we have $1-r \cos \theta \leq 1-r+r c \theta^{2}$. Hence (49) follows from the remark on (50).

(ii) Assume $|\theta| / 2 \leq|\tau|$, and set $t:=\tau /(1-r)$. For the left hand side of (49) we simply use the lower bound $1-r$. The right hand side is bounded by

$$
\begin{aligned}
\frac{1}{C^{1 / \beta}} t^{-a / \beta}(1-r \cos \theta) & \leq \frac{1}{C^{1 / \beta}} t^{-a / \beta}\left(1-r\left(1-4 c t^{2}(1-r)^{2}\right)\right) \\
& \leq \frac{1}{C^{1 / \beta}} t^{-a / \beta}\left(1-r+4 c t^{2}(1-r)^{2}\right) \\
& =\frac{1}{C^{1 / \beta}} t^{-a / \beta}(1-r)+\frac{4 c}{C^{1 / \beta}} t^{-a / \beta+2}(1-r)^{2} .
\end{aligned}
$$

Both terms in the last line can be bounded by $(1-r) / 3$ by choosing $a \geq 2 \beta$ and $C$ large enough.

Because of the rotation invariance of the conditions involved, also weights of the form

$$
v(z):=\left(1-|z|^{2}\right)^{\alpha}\left(1-\left(\operatorname{Re}\left(e^{i \varrho} z\right)\right)^{2}\right)^{\beta}, \quad \varrho \in[-\pi, \pi],
$$


satisfy the conditions of Proposition 2. One can then go on by multiplying weights corresponding to different @'s (see Proposition 3).

EXAMPLE 4. Wolfgang Lusky proved in [8] that the Riesz projection (i.e. Szegö projection) is bounded from

$$
h_{v}^{\infty}:=\left\{f: \mathbb{D} \rightarrow \mathbb{C} \text { harmonic } \mid\|f\|_{v}<\infty\right\}
$$

onto $H_{v}^{\infty}$ if the radial weight $v$ is decreasing as $|z|$ increases and satisfies

$$
\sup _{N \in \mathbb{N}} \sum_{k=1}^{\infty}\left(1-\frac{1}{2^{N+1}}\right)^{2^{N+k}} \frac{v\left(1-2^{-N}\right)}{v\left(1-2^{-N-k}\right)}<\infty
$$

and

$$
\inf _{k \in \mathbb{N}} \limsup _{N \rightarrow \infty} \frac{v\left(1-2^{-N-k}\right)}{v\left(1-2^{-N}\right)}<1
$$

Let us show that such a weight $v$ satisfies (25) and (26). Actually (25) follows from the facts that $v$ is radial and decreasing, since (54) implies $v\left(1-2^{-N}\right) \leq C v\left(1-2^{-N-1}\right)$.

Concerning the latter condition, since $v$ is radial, we have $v\left(\lambda_{n, m}\right)=$ $v\left(\lambda_{n, 2^{n}}\right)=v\left(1-2^{-n}\right)$, by the choice of $\lambda_{n, m}$ (see (23)). Hence, the left hand side of (26) equals

$$
\begin{aligned}
\sum_{n<N, m \in M_{n}} \frac{1}{\mu^{2+\alpha}} \frac{1}{v\left(1-2^{-n}\right)}+\sum_{n \geq N} \sum_{m: \mu \leq 2^{n-N}} \frac{1}{v\left(1-2^{-n}\right)} 2^{(-n+N)(2+\alpha)} \\
\quad+\sum_{n \geq N} \sum_{m: 2^{n-N} \leq \mu \leq 2^{n}} \frac{1}{\mu^{2+\alpha}} \frac{1}{v\left(1-2^{-n}\right)} \\
\leq \sum_{n<N} \frac{1}{v\left(1-2^{-n}\right)}+\sum_{n \geq N} \frac{1}{v\left(1-2^{-n}\right)} 2^{(-n+N)(2+\alpha)+n-N} \\
+\sum_{n \geq N} \frac{1}{v\left(1-2^{-n}\right)} 2^{(\alpha+1)(-n+N)} \\
=I+I I+I I I,
\end{aligned}
$$

where in the last term we used $\sum_{m: \mu \geq 2^{n-N}} \mu^{-2-\alpha} \leq C 2^{(\alpha+1)(-n+N)}$. Clearly, $I I=I I I$. Now (55) implies that there exists a $c, 0<c<1$, such that for all $n<N$,

$$
v\left(1-2^{-n}\right) \leq C c^{N-n} v\left(1-2^{-N}\right) .
$$

Hence the term $I$ is bounded by

$$
\sum_{n<N} \frac{1}{v\left(1-2^{-n}\right)} \leq \sum_{n<N} \frac{C c^{n-N}}{v\left(1-2^{-N}\right)} \leq \frac{C^{\prime}}{v\left(1-2^{-N}\right)} .
$$


Moreover, (54) implies

$$
\sum_{k=1}^{\infty}\left(1-\frac{1}{2^{N+1}}\right)^{2^{N+k}} \frac{1}{v\left(1-2^{-N-k}\right)} \leq \frac{C}{v\left(1-2^{-N}\right)},
$$

yielding

$$
\sum_{n>N}((1+\varepsilon) e)^{-n+N} \frac{1}{v\left(1-2^{-n}\right)} \leq \frac{C_{\varepsilon}}{v\left(1-2^{-N}\right)}
$$

for any $\varepsilon>0$. So, if $\alpha$ is so large that $2^{\alpha+1} \geq e$, then (57) implies $I I \leq$ $C v\left(1-2^{-N}\right)$, completing the proof of $(26)$.

4. Logarithmic case. If the radial weight $v$ approaches 0 only at logarithmic speed, there do not exist any bounded projections from $L_{v}^{\infty}$ onto $H_{v}^{\infty}$. See for example [10]. If any projection operator $P$ on such an $L_{v}^{\infty}$ is given, one can find $f$ belonging to the unit ball of $L_{v}^{\infty}$ such that $\sup _{|z|=r}|P f(z)|$ behaves, heuristically speaking, like $|\log (1-r)| / v(r)$ (or worse) as $r \rightarrow 1$, i.e. $P$ makes the function $f$ worse by a logarithmic factor.

Something can still be done for the projective description problem for holomorphic inductive limits. For the sake of comparison, cut-off arguments form a basic tool for that problem in the case of continuous functions. Of course, for analytic functions such methods are excluded. One can however try to use a cut-off argument inside the Bergman projection, i.e. apply a cut-off to an analytic function and then operate by Bergman projection. So let us look at that. Given an analytic $f$ we make a formal calculation using a nonsmooth partition of unity $\sum_{n} \varphi_{n}$ with $\varphi_{n}(z)=1$ if and only if $r_{n}<|z| \leq r_{n+1}$ for some numbers $\left.r_{n} \in\right] 0,1[$ :

$$
\begin{aligned}
f(z) & =\int_{\mathbb{D}} \frac{\sum_{n} \varphi_{n}(\zeta) f(\zeta)}{(1-z \bar{\zeta})^{2}} d A(\zeta)=\sum_{n=1}^{\infty} \int_{r_{n}}^{r_{n}+1} \int_{0}^{2 \pi} \frac{f\left(r e^{i \theta}\right)}{\left(1-z r e^{-i \theta}\right)^{2}} r d r d \theta \\
& =\sum_{n=1}^{\infty} \int_{r_{n}}^{r_{n+1}}\left(\int_{0}^{2 \pi} \frac{e^{i \theta} f\left(r e^{i \theta}\right)}{\left(e^{i \theta}-z r\right)^{2}} e^{i \theta} d \theta\right) r d r \\
& =C \sum_{n=1}^{\infty} \int_{r_{n}}^{r_{n+1}}\left[\frac{\partial}{\partial w}(r w f(r w))\right]_{w=r z} d r \\
& =C \frac{1}{z} \sum_{n=1}^{\infty} \int_{z r_{n}}^{z r_{n+1}} \frac{\partial}{\partial w}\left(\frac{w^{2}}{z} f\left(\frac{w^{2}}{z}\right)\right) d w \\
& =C \sum_{n=1}^{\infty} r_{n+1}^{2} f\left(r_{n+1}^{2} z\right)-r_{n}^{2} f\left(r_{n}^{2} z\right) .
\end{aligned}
$$


It turns out that the representation (58) is useful if the weights tend to zero logarithmically on the boundary and the points $r_{n}$ are properly chosen. We will actually use a simplified representation, omitting the extra coefficients $r_{n}^{2}$. We will not explicitly use the Bergman projection, but the hidden connection with it is explained in (58). For every $n \in \mathbb{N}$ we set

$$
r_{n}:=1-2^{-2^{n}}, \quad r_{0}=0, \quad I_{n}:=\left[r_{n}, r_{n+1}\right] .
$$

In this section we only consider the case of radial weights which approach monotonically 0 as $r \rightarrow 1^{-}$.

DEFinition 1. We say that the weight sequence $\mathcal{V}$ satisfies the condition (LOG) if there exist constants $0<a<1<A$ such that

$$
A v_{k}\left(r_{n+1}\right) \geq v_{k}\left(r_{n}\right)
$$

and

$$
v_{k}\left(r_{n+1}\right) \leq a v_{k}\left(r_{n}\right)
$$

for all $k$ and $n$.

The first condition is an upper bound for the speed with which the weights approach zero on the boundary, and the second condition is a lower bound.

EXAMPLE 5. It is clear that a weight $v(z):=(-1 / \log (1-|z|))^{\alpha}$, where $\alpha>0$, satisfies both (60) and (61). Interesting weight families $\mathcal{V}$ satisfying (LOG) are those of the type

$$
v_{k}(z)=(-1 / \log (1-|z|))^{\alpha(k,|z|)},
$$

where $0<c \leq \alpha(k,|z|) \leq C$ for all $k$ and $z$. For any fixed $k$, the function $\alpha(k, r)$ must not vary too quickly with respect to $r$ in order that (LOG) be satisfied. We leave the details to the reader.

EXAMPLE 6. Let us describe another way of finding many interesting weight systems satisfying (LOG).

Suppose that $\mathcal{W}=\left(w_{k}\right)_{k=1}^{\infty}$ is an arbitrary decreasing sequence of weights on $\mathbb{D}$. We only assume that there exists a strictly increasing sequence $\left(s_{n}\right)_{n=1}^{\infty}$ $\subset] 0,1[$ such that, for some $C>1$,

$$
C w_{k}\left(s_{n+1}\right) \geq w_{k}\left(s_{n}\right)
$$

for all $k$ and $n$. (Notice that this condition does not restrict the behaviour of a single weight; it is just a very mild restriction on the whole weight family.)

We claim that one can define a weight sequence $\mathcal{V}=\left(v_{k}\right)_{k=1}^{\infty}$ satisfying (LOG) such that $v_{k}$ is just of the form

$$
v_{k}(z):=\psi(|z|) w_{k}(\varphi(|z|)),
$$


where $\psi:\left[0,1\left[\rightarrow \mathbb{R}^{+}\right.\right.$and $\varphi:[0,1[\rightarrow[0,1[$ are chosen suitably. It is clear from the definition (64) that $\mathcal{V}$ satisfies for example the condition $(D),(R D)$ etc. (for definitions, see [3]) if and only if the original sequence $\mathcal{W}$ does. As a consequence (of Theorem 5 below) we find that these conditions are not relevant (not needed) for the projective description in the case of spaces with 0 -subindex (o-growth conditions).

To find the functions $\psi$ and $\varphi$, let $r_{n}$ be as above, and choose $\varphi\left(r_{n}\right):=s_{n}$ for every $n$. Extend $\varphi$ piecewise linearly to $[0,1[$. Finally, take $\psi(r):=$ $(-1 / \log (1-r))$. Define every $v_{k}$ by $(64)$.

We have $2 \psi\left(r_{n+1}\right)=\psi\left(r_{n}\right)$ for every $n$, hence (60) follows for every $v_{k}$ from (63). Moreover,

$$
v_{k}\left(r_{n+1}\right)=\psi\left(r_{n+1}\right) w_{k}\left(s_{n+1}\right) \leq \frac{1}{2} \psi\left(r_{n}\right) w_{k}\left(s_{n}\right)=\frac{1}{2} v_{k}\left(r_{n}\right),
$$

hence also (61) is satisfied.

THEOREM 5. If the weight system $\mathcal{V}$ satisfies the condition (LOG), then $\mathcal{V}_{0} H(\mathbb{D})$ is a topological subspace of $H \bar{V}_{0}(\mathbb{D})$.

Proof. For every $k \in \mathbb{N}$, denote by $U_{k}$ the set of those functions $f \in$ $H\left(v_{k}\right)_{0}(\mathbb{D})$ for which $\|f\|_{v_{k}} \leq 1$. Let a 0-neighbourhood $B:=\Gamma\left(\bigcup_{k=1}^{\infty} b_{k} U_{k}\right)$ in $\mathcal{V}_{0} H$ be given, where $\Gamma$ stands for the absolutely convex hull and $b_{k}>0$ for every $k$. Let us define the decreasing weight

$$
\bar{v}(z):=\inf _{k \in \mathbb{N}} a_{k}^{-1} v_{k}(z),
$$

with $a_{k}<2^{-k-1} A^{-2} b_{k}$, with $A$ as in (60), and such that $\bar{v}$ is radial, continuous, nonincreasing and the infimum is a minimum on compact subsets of $\mathbb{D}$; see [5]. Assuming that $f \in \mathcal{V}_{0} H$ satisfies $\|f\|_{\bar{v}} \leq 1$, we show that $f \in \bar{B}$, closure taken in $\mathcal{V}_{0} H$. It is known that any $g \in \mathcal{V}_{0} H$ can be approximated arbitrarily well in $\mathcal{V}_{0} H$ by the functions $g_{r_{n}}(z):=g\left(r_{n} z\right)$ for large $n$. (Taking a $k$ such that $g \in\left(H v_{k}\right)_{0}$ we have $\lim _{|z| \rightarrow 1^{-}}|g(z)| v_{k}(z)=0$. This implies $g_{r_{n}} \rightarrow g$ in $\left(H v_{k}\right)_{0}$ as $n \rightarrow \infty$. The topology of the inductive limit is coarser, hence, $g_{r_{n}} \rightarrow g$ also there.) Hence, it suffices to show $f_{r_{\nu}} \in B$ for every large enough $\nu \in \mathbb{N}$.

By (60), since the weight $\bar{v}$ is nonincreasing, we get

$$
\inf _{|z| \in I_{n}} \bar{v}(z)=\bar{v}\left(r_{n+1}\right) \geq \bar{v}\left(r_{n+2}\right)=\inf _{|z| \in I_{n+1}} \bar{v}(z) \geq A^{-2} \bar{v}\left(r_{n}\right) .
$$

For every $n$ we can thus pick a $k(n) \in \mathbb{N}$ such that

$$
\bar{v}\left(r_{n}\right)=a_{k(n)}^{-1} v_{k(n)}\left(r_{n}\right)=a_{k(n)}^{-1} \sup _{|z| \in I_{n}} v_{k(n)}(z) .
$$

Fix $\nu \in \mathbb{N}$, and for every $m$, let $N_{m} \subset \mathbb{N}$ be $N_{m}:=\{n \leq \nu \mid k(n)=m\}$. Hence, the set $\{n \leq \nu \mid n \in \mathbb{N}\}$ is a disjoint union of the sets $N_{m}$; some of them may be empty sets. Let us define, for all $n$, the function $g_{n}(z):=$ 
$f\left(r_{n+1} z\right)-f\left(r_{n} z\right)$, and $g_{0}(z):=f(0)$, and, for all $m$,

$$
h_{m}:=\sum_{n \in N_{m}} g_{n}
$$

and $h_{m}:=0$ if $N_{m}=\emptyset$. Clearly $f_{r_{\nu}}=\sum_{m \in \mathbb{N}} h_{m}+g_{0}$. The constant function $g_{0}$ belongs to $H\left(v_{k(0)}\right)_{0}$, and $|f(0)| \leq a_{k(0)} v_{k(0)}(0)^{-1}$, hence $g_{0} \in\left(b_{m} / 2^{m}\right) U_{m}$ for $m=k(0)$. The main part of the proof is to show the following

Lemma 2. If $C>0$ satisfies $C>\sum_{k \in \mathbb{N}} a^{k}$ with $0<a<1$ as in (61) and $C>\left(2 A^{t} 2^{n-t}\right) / 2^{2^{n}}$ if $n>t$, then $h_{m} \in C 2^{-m} b_{m} U_{m}$ for all $m$.

This implies the theorem: since $h_{m} \in C 2^{-m} b_{m} U_{m}$, we have

$$
f_{r_{\nu}}=\sum_{m \in \mathbb{N}} h_{m}+g_{0} \in 2 C \Gamma\left(\bigcup_{m \in \mathbb{N}} b_{m} U_{m}\right) \text {. }
$$

Proof of Lemma 2. Fix an $m \in \mathbb{N}$. We pick an $n \in N_{m}$ and estimate $\left|g_{n}(z)\right|$ for different $z$.

$1^{\circ}$. Assume first $|z| \geq r_{n-1}$. Then

$$
\left|r_{n} z\right| \geq\left(1-2^{-2^{n}}\right)\left(1-2^{-2^{n-1}}\right) \geq\left(1-2 \cdot 2^{-2^{n-1}}\right) \in I_{n-2},
$$

and similarly for $\left|r_{n+1} z\right|$; hence

$$
r_{n-2} \leq\left|r_{n} z\right| \leq\left|r_{n+1} z\right| \leq r_{n+1} .
$$

Since $\|f\|_{\bar{v}} \leq 1$, we have for these $z$, by (66),

$$
\left|g_{n}(z) \leq\right| f\left(r_{n} z\right)|+| f\left(r_{n+1} z\right) \mid \leq 2 \sup _{r_{n-2} \leq r \leq r_{n+1}} \bar{v}(r)^{-1}=2 \bar{v}\left(r_{n+1}\right)^{-1} .
$$

Now (70) can still be estimated using (67) by

$$
2 A^{2} \bar{v}\left(r_{n}\right)^{-1}=2 A^{2} a_{m} v_{k(n)}\left(r_{n}\right)^{-1} \leq 2^{-m} b_{m} v_{m}\left(r_{n}\right)^{-1},
$$

by the choice of $a_{k(n)}=a_{m}$.

$2^{\circ}$. Assume $2 \leq t \leq n$ and $|z| \in I_{n-t}$. We have

$$
\begin{aligned}
\left|g_{n}(z)\right| & =\left|f\left(r_{n} z\right)-f\left(r_{n+1} z\right)\right| \\
& \leq \sup _{\zeta \in I_{n-t} \cup I_{n-t-1}}\left|f^{\prime}(\zeta)\right|\left|r_{n+1}-r_{n}\right| \leq \sup _{\zeta \in I_{n-t} \cup I_{n-t-1}}\left|f^{\prime}(\zeta)\right| 2^{-2^{n}}
\end{aligned}
$$

We estimate $\left|f^{\prime}(\zeta)\right|$ using the Cauchy formula:

$$
\left|f^{\prime}(\zeta)\right| \leq \int_{|\eta|=r_{n}} \frac{|f(\eta)|}{|\eta-\zeta|^{2}} d \eta \leq \bar{v}\left(r_{n}\right)^{-1} 2^{2^{n-t+1}},
$$

since $|\eta-\zeta| \geq 2^{-2^{n-t+1}}-2^{-2^{n}} \geq 2^{-1} \cdot 2^{-2^{n-t+1}}$. We use $2^{n}-2^{n-t+1} \geq 2^{n-1}$, and from (72) and (73) we obtain

$$
\left|g_{n}(z)\right| \leq 2^{-2^{n-1}} \cdot \bar{v}\left(r_{n}\right)^{-1} \leq 2^{-2^{n-1}} \cdot a_{k(n)} v_{k(n)}\left(r_{n}\right)^{-1}
$$


Here we used (67). Moreover, using (60) $t$ times, we can continue the estimate by

$$
\leq 2^{-2^{n-1}} \cdot A^{2} a_{k(n)} v_{k(n)}(z)^{-1} .
$$

Since $n>t$, we have $2^{-2^{n}} A^{t} \leq C 2^{-(n-t)}$ (for all $n$ and $t$ ), hence, (75) is bounded by

$$
C 2^{-(n-t)-k(n)} b_{k(n)} v_{k(n)}(z)^{-1}=C 2^{-(n-t)-m} b_{m} v_{m}(z)^{-1} .
$$

So altogether,

$$
\left|g_{n}(z)\right| \leq C 2^{-(n-t)-m} b_{m} v_{m}(z)^{-1} .
$$

To complete the proof of the lemma, let now $z \in \mathbb{D}$; we want to show that

$$
\left|h_{m}(z)\right| \leq C 2^{-m} b_{m} v_{m}(z)^{-1} .
$$

Let $t \in \mathbb{N}$ be such that $|z| \in I_{t}$; then

$$
\left|h_{m}(z)\right| \leq \sum_{\substack{n \in N_{m} \\ n \leq t+1}}\left|g_{n}(z)\right|+\sum_{\substack{n \in N_{m} \\ n>t+1}}\left|g_{n}(z)\right|=: G_{m}(z)+H_{m}(z) .
$$

(a) Consider $G_{m}(z)$. In this case (71) of $1^{\circ}$ implies

$$
G_{m}(z) \leq \sum_{\substack{n \in N_{m} \\ n \leq t+1}} 2^{-m} b_{m} v_{m}\left(r_{n}\right)^{-1}
$$

By using (61) $t-n$ times, we see that this is bounded by a constant times

$$
\sum_{n \leq t+1} 2^{-m} b_{m} v_{m}\left(r_{t}\right)^{-1} a^{-t+n} \leq C 2^{-m} b_{m} v_{m}(z)^{-1} .
$$

Remember that $a<1$ and $C>\sum_{k \in \mathbb{N}} a^{k}$.

(b) Consider $H_{m}(z)$. Then (76) implies

$$
H_{m}(z) \leq \sum_{\substack{n \in N_{m} \\ n>t+1}} C b_{m} v_{m}(z)^{-1} 2^{-m} 2^{-n+t} \leq C b_{m} v_{m}(z)^{-1} 2^{-m}
$$

We get (77), and thus the lemma, from (79) and (80).

\section{References}

[1] H. Bateman and A. Erdélyi, Higher Transcendental Functions II, McGraw-Hill, 1953.

[2] D. Békollé, Inégalités à poids pour le projecteur de Bergman dans la boule unité de $\mathbf{C}^{n}$, Studia Math. 71 (1982), 305-323.

[3] K. D. Bierstedt, A survey of some results and open problems in weighted inductive limits and projective description for spaces of holomorphic functions, Bull. Soc. Roy. Sci. Liège 70 (2001), 167-182. 
[4] K. D. Bierstedt and J. Bonet, Projective description of weighted (LF)-spaces of holomorphic functions on the disc, Proc. Edinburgh Math. Soc. 46 (2003), 435-450.

[5] K. D. Bierstedt, R. Meise and W. Summers, A projective description of weighted inductive limits, Trans. Amer. Math. Soc. 272 (1982), 107-160.

[6] J. Bonet and J. Taskinen, Subspace problem for weighted inductive limits, Michigan Math. J. 42 (1995), 259-268.

[7] F. Holland and R. Rochberg, Bergman kernel asymptotics for generalized Fock spaces, J. Anal. Math. 83 (2001), 207-242

[8] W. Lusky, On weighted spaces of harmonic and holomorphic functions, J. London Math. Soc. 51 (1995), 309-320.

[9] P. Mattila, E. Saksman and J. Taskinen, Weighted spaces of harmonic and holomorphic functions: sequence space representations and projective descriptions, Proc. Edinburgh Math. Soc. 40 (1997), 41-62.

[10] J. Taskinen, On the continuity of Bergman and Szegö projections, Houston J. Math. 30 (2004), 171-190.

[11] K. Zhu, Operator Theory in Function Spaces, Dekker, New York, 1995.

E.T.S. Arquitectura

MÚ AV ČR

Departamento de Matemática Aplicada

Žitná 25

Universidad Politécnica de Valencia

E-46071 Valencia, Spain

11567 Praha 1, Czech Republic

E-mail: jbonet@mat.upv.es

E-mail: englis@math.cas.cz

Department of Mathematics and Statistics

University of Helsinki

P.O. Box 68

FIN-00014 Helsinki, Finland

E-mail: jari.taskinen@helsinki.fi

Received November 29, 2004

Revised version April 26, 2005 